\title{
ZEILEN AUS DEM GRAB. DIE STRUKTUR DER FRÜHEN RADNÓTI-REZEPTION
}

\author{
GÁBOR SCHEIN
}

Eötvös Loránd University
gaborschein@hotmail.com

Für die kultische, bzw. hagiographische frühe Rezeption der Dichtung von Miklós Radnóti erwies sich jene Stelle bei Abda unter dem Damm des Flusses Rábca, wo er mit 21 anderen jüdischen Zwangsarbeitern Ende August 1944 von einem ungarischen Soldaten erschossen wurde, als ein bindender Ort. Nachdem der Körper des Dichters in einem Massengrab mit anderen Leichen verscharrt worden war, wurde er noch zweimal exhumiert und neu bestattet. Der traumatische Ort des Mordes schreibt sich durch die letzten Gedichte ins Gedächtnis der Generationen. Die traumatisierte frühe Rezeption von Radnóti besteht auf den Stillstand der Zeit, auf die Nähe zum mehrmals beigesetzten und wieder ausgegrabenen Körper des Dichters. Diese seltsame Art der Rezeption, die die Dichtung von Radnóti der Historisierung zu entziehen versucht, ist als eine Antwort auf die Frage zu verstehen, ob der Holocaust in seiner Außergeschichtlichkeit zu bewahren ist. In der Ästhetik der Zeugenschaft wiederholt sich die Frage, wie man eine einmalige Raum-Zeit-Beziehung in ihrer Form bewahren kann, die sowohl das Trauma der Zerstörung einer historischen Gemeinschaft, der Gemeinschaft der ungarischen Nation als auch die Hoffnung auf eine Therapie, auf eine Neustiftung der Gemeinschaft miteinschließt. Das Gedicht von Radnóti „Nem tudhatom“ stiftete in der frühen Rezeption eine neue Perspektive für die Neubelebung der durch den Holocaust zerrissenen Gemeinschaft der Nation, es konnte aber von der romantischen Ideologie der nationalen Identität nicht Abschied nehmen, und dadurch blieb diese neue Perspektive unvollendet.

Schlüsselwörter: Miklós Radnóti, Holocaust, Rezeptionsgeschichte, Ästhetik der Zeugenschaft

Beerdigung und Ausgrabung erweisen sich seit Freud als Grundmetapher der Konzeptualisierung der Psychoanalyse. In seinem Aufsatz „Konstruktionen in der Analyse" hat Freud die Methode des Analytikers mit der eines Archäologen in eine Parallele gestellt. Ausgraben heißt bei ihm die konstruktive Verbundenheit an das einmal Gewesene, die die passive Macht der Verschüttung und der Zer- 
trümmerung durch Ergänzung und Zusammenfügung, d.h. durch das Vorgehen in einer ganz spezifischen Hermeneutik, beseitigt. Sein Optimismus beruht nicht zuletzt auf einer idealisierten Qualität des Verschütteten, die die Möglichkeit in sich trägt, durch die Leistung des archäologisch vorgehenden Analytikers ein dem Einstigen entsprechendes Ganzes wiederbeleben zu können. In diesem Sinne verhalten sich alle Reste als kulturelle Gedächtnismaschinen, als Speicherungseinheiten, die die Identität der Einlagerung und Rückholung ${ }^{1}$ zum Ziel haben: „Wie der Archäologe an stehengebliebenen Mauerresten die Wandlungen des Gebäudes aufbaut, aus Vertiefungen im Boden die Anzahl und Stellung der Säulen bestimmt, aus den im Schutt gefundenen Resten die einstigen Wandverzierungen und Wandgemälde wiederherstellt, genauso geht der Analytiker vor, wenn er seine Schlüsse aus Erinnerungsbrocken, Assoziationen und aktiven Äußerungen des Analysierten zieht.“2

Das Ausgraben macht die Reste des verschütteten und einst gegebenen Sachverhaltes offenkundig, und damit öffnet es sie für das Neu-Beleben. Im Sinne von Freud ist es ein singuläres Ereignis, ein Akt des konzipierenden-wiederherstellenden Gedächtnisses, das ihren Grund darin findet, dass die Vergangenheit nicht völlig und restlos vergehen kann. Für Walter Benjamin ist die Arbeit des Gedächtnisses kein Akt. Sein Wesen äußert sich bei ihm eher in einer meditativ-reflexiven Einstellung, die sich nicht auf die Beseitigung der Verschüttung richtet, die einen aber immer wieder zum Verschütteten zurückkehren, zu diesem zurückwenden lässt. „Wer sich der eigenen verschütteten Vergangenheit zu nähern trachtet, muß sich verhalten wie ein Mann, der gräbt. Vor allem darf er sich nicht scheuen, immer wieder auf einen und denselben Sachverhalt zurückzukommen - ihn auszustreuen, wie man Erde ausstreut, ihn umzuwühlen, wie man Erdreich umwühlt." ${ }^{\text {3 }}$ Die Erde wird bei Benjamin zum Medium des Verschütteten, das auf einen bestimmten Ort, auf den Ort der Ausgrabung der Erinnerung verweist. Erinnerungen sind also verortet, d.h. sie haben keinen objektiven Charakter, indem sie sich niemals vom Milieu der Ausgrabung vollständig ablösen lassen. ${ }^{4}$

Um so stärker noch ist die Bindungskraft des Ortes, wenn ein Ausgraben mit der Unwiederherstellbarkeit eines gewaltsam geraubten Lebens konfrontiert. Der archäologische Optimusmus von Freud scheitert in diesem Falle am Bruch zwischen dem unvermittelbaren Ereignis und dem unwiderruflich vernichteten Leben. Für die kultische bzw. hagiographische Rezeption der Dichtung von Miklós Radnóti erweist sich jene Stelle bei Abda, unter dem Damm des Flusses Rábca, mitten in einem kleinen Birkenwald, wo er zusammen mit 21 anderen jüdischen Zwangsarbeitern Ende August 1944 von einem ungarischen Soldaten erschossen wurde, als ein bindender Ort. Der Todesweg, der vom serbischen Bor ausgehend durch Cservenka, Mohács und Szentkirályszabadja von Süden nach Norden ganz Ungarn durchkreuzt, wurde nach der Entdeckung der letzten Gedichte, die diesen Weg durch ihre Datierungen genau beschrieben, als eine Art Via Dolorosa des 
Dichter-Märtyrers konzipiert. Beerdigung und Ausgrabung sind an diesem Ort keine Metapher mehr, keine hermeneutische Paralelle, sondern wirkliche Maßnahmen des kulturellen Gedächtnisses.

Als der Todesschuss den Schädel des Dichters durchschlug, fiel dessen Körper in ein Massengrab. Dieses Massengrab wurde nach dem Ende des Krieges freigelegt, und die 22 Opfer wurden im jüdischen Friedhof von Raab beigesetzt. Während der Freilegung kam es zu der Identifizierung mehrerer Toten. Die Witwe und die Freunde des Dichters durften erst am 12. August 1946 an der zweiten Exhumierung des Leichnams teilnehmen. Gyula Otutay, einer der ganz engen Freunde des Dichters, notierte von diesem Tag in seinem Tagebuch: „Im Sarg flatterten schon Fliegen, und der blutige, missgeformte Rest, den wir sahen und der uns ekelte, war irgendwie so wenig, so furchtbar unbedeutend und nichtig, dass es nicht an den Tod erinnerte, sondern an eine Art von beschämendem, schändlichem Mörtel, der weder mit dem Tod noch mit dem Leben, besonders nicht mit jenem von Miklós, zu tun hat, es war nur eine zerfallende, fremde und ekelhafte Materie, dreckiger Schlamm, eine blutige Anschwemmung." “5

Die Beschreibung von Ortutay wird von Metaphern (Schlamm, Anschwemmung) gesteuert, die aus direkter Nähe des Todesortes von Radnóti, d.h. aus dem Milieu des primären Gedächtnisses stammen. Die ekelhafte Wirklichkeit der zerfallenden Leiche setzt einen textualen Prozess in Gang, der das Gedächtnis durch die kognitive Leistung der Metapher zum Todesort zurückführt. Die Rückkehr zum Todesort, die Rückwendung zum gewaltsamen Tod, zur Hinrichtung ist die grundlegende, sich zwanghaft wiederholende Bewegung in der Rezeption von Miklós Radnóti. Die Zeit steht in der Lektüre seiner letzten Gedichte still, und weil man am Todesort immer wieder den gewaltsamen Abbruch eines Lebens erlebt, wird das Gedächtnis in dieser stillstehenden Zeit, bzw. in der traumatisierten Zeitlichkeit, letzten Endes von der Erde, die einst den Körper in sich aufnahm, angesprochen und herausgefordert. Die Erde ist das Medium der Erinnerung und der konstruktive Widerpart der Lektüre.

Der Todesort von Miklós Radnóti wurde im Lauf der Jahrzehnte nach der Exhumierung ein Gedenkort und eine Kultstätte. Sie markiert im tiefsten Sinne des Wortes Diskontinuität. Das Anwesende, die im Jahre 1960 enthüllte Büste von László Alexovits, verweist in erster Linie nicht auf die Abwesenheit der abgebildeten Person, sondern auf den Mord. Der traumatische Ort des Mordes schreibt sich ins Gedächtnis der Generationen durch die letzten Gedichte, deren Entdeckung wir der Exhumierung zu verdanken haben. Das Notizheft, das sogenannte „Notizheft von Bor“, das diese letzten Gedichte enthielt, übernahm die Witwe am jüdischen Friedhof von Raab. Es war in einem Tresor der jüdischen Gemeinde aufbewahrt worden. Ortutay vermeinte sich aber mehr als zehn Jahre später zu erinnern, dass er das Notizheft unter den blutigen, zerfetzten Kleidungsstücken des Dichters im Sarg aufgefunden habe. ${ }^{6}$ Die Szene verschob sich somit in sei- 
ner Erinnerung, er hatte die direkte Verbindung zwischen den letzten Gedichten und der Leiche wiederhergestellt. Mit dieser Transformation der Vergangenheit hat er weniger der Ästhetisierung als vielmehr der Historisierung der letzten Gedichte Einhalt geboten. Ihre traumatisierte Rezeption besteht auf den Stillstand der Zeit, besteht auf die Nähe zu diesem mehrmals beigesetzten und ausgegrabenen Körper des Dichters. Diese Nähe verortet die Lektüre, die sich dadurch in der Zeugenschaft beweist. In der Ästhetik der Zeugenschaft wiederholt sich die Frage, wie man eine einmalige Raum-Zeit-Beziehung in einer Form behalten kann, die sowohl das Trauma der Zerstörung einer historischen Gemeinschaft, der Gemeinschaft der ungarischen Nation, als auch die Hoffnung auf eine Therapie, auf eine Neustiftung der Gemeinschaft mit einschließt. Es ist kein Zufall, dass László Bóka, ein einflussreicher Literaturhistoriker jener Zeit, in einer Rezension 7 über den posthum erschienenen Band „Gischthimmel“ (Tajtékos ég) im Jahre 1946 Radnóti einen „Nationaldichter“ genannt hat. Die Rezeption ist zwar ein geschichtliches Kontinuum, in diesem Fall aber entfaltet sie einen Kult, der der Historisierung widersteht, und immer wieder zum Tod des Dichters wiederkehrt. Im Ritual des Todes hat die Todeswache eine ähnliche Funktion. Die erste Phase der kultischen Rezeption endete am 14. August 1946, als der zerfallende Körper von Miklós Radnóti am Friedhof an der Fiumei-Straße in Budapest, diesmal unter katholischer Liturgie, endgültig beigesetzt wurde. Die Trauerreden zitierten die Gedichte von Radnóti als Epitaphien, wobei sich die traumatischen Gefühle nicht mildern ließen. Mehrere sprachen am Grab über Scham und Schuldbewusstsein.

Nach dem Abschluss der Phase der Exhumierungen und Beerdigungen verblieb die Steuerung der Rezeption noch über lange Zeit in der Hand des engeren Freundeskreises des Dichters, und in ihrem Hintergrund stand die in mehreren Gedichten angesprochene Witwe, die während ihres besonders langen Lebens - unlängst feierte sie ihren 97. Geburtstag - immer als Inbegriff der liebevollen Treue zu dem verstorbenen Dichter zählte. Ihre Seele schwebte auch über dem Jubiläumsjahr 2009, als Ungarn den 100 Jahre zuvor geborenen Dichter feierte, einem Jubiläum, dessen Höhepunkt in der Nacht von Radnótis Geburtstags eine seltsame Wache war, ein nächtlicher Anlass, zu dem in Budapest eine Reihe von Veranstaltungen wie Lesungen, Ausstellungen und Gespräche dem Andenken des Dichters Tribut zollten.

Diese seltsame Art der Rezeption, die sich auf den Stillstand der Zeit richtet, in der sich die Dichtung von Radnóti der Historisierung zu entziehen versucht, ist als eine Antwort auf die Frage zu verstehen, ob der Holocaust in seiner Außergeschichtlichkeit zu bewahren ist. Der Holocaust ist ein historisches Ereignis, das vor einer sprachpolitisch dienstbar gemachten Metaphorisierung unbegrenzbar offen steht, mithin lässt es sich als sprachliches Modell der Vergangenheitsbewältigung leicht in Sprachspiele transformieren, wobei es jedoch stets in der peinlichen, provokativen Realität des „Nicht-Verstanden-Habens“ steckenbleibt. 
Diese außerordentliche sprachliche Unausgewogenheit, die ein so augenfälliger Charakterzug der Rede über den Holocaust ist, erhält das, was geschehen ist, tatsächlich im metahistorischen Sinne in der Gegenwart, wobei sie von der Gegenwart eine Entscheidung erfordert: Sind wir denn imstande, ihn in einer Außergeschichtlichkeit zu bewahren, oder setzen wir die unumgängliche und unabwendbare Erfahrung als bloßen Unfall, als abrupten Störfall im ordentlichen Gang der Dinge in die Geschichte zurück?

Die Raum-Zeit-Struktur der frühen Radnóti-Rezeption, die die unbedingte Nähe des Todes und des Toten suchte, verschränkte Präsenz mit Absenz, sinnliche Gegenwart mit Vergangenheit. In diesem Sinne war sie nicht nur mit einem Trauma beladen, sie bot auch eine therapeutische Stütze für die Überlebenden, für eine gebrochene Gemeinschaft, die über den Holocaust nicht hinausschauen konnte, weil sie sich auf allen Seiten des Rituals bediente, viel weniger des Realismus. Realismus wäre hier natürlich nicht mit der Tendenz alles zu de-ästhetisieren zu verwechseln. Es hat nichts mit dem Verlangen von Adorno zu tun, der sich bekanntermaßen weigerte, den Künsten bei der Trauer über die Vernichtung eine Rolle zuzubilligen. Realismus meint hier einfach die Kenntnisnahme und das Erkennen des Geschehenen in seiner Faktizität. Gibt es eine in diesem Sinne wirkliche und wirksame Therapie ohne Realismus? Die Dichtung von Radnóti leistete mindestens zwei wichtige Beiträge zu einer realitätsentzogenen Bewältigung der traumatischen Vergangenheit. Wenn wir aber dabei sind, können wir einer der wichtigsten Fragen der frühen Radnóti-Rezeption nicht entkommen: Inwieweit ließe sich das Gesicht, das diese Rezeption formte, auf die subjektbildenden Verfahren dieser Lyrik in der tropologischen Dimension zurückführen?

Die frühe Rezeption entfaltete sich in einem Begriffsrahmen, der seine Bestimmung vor allem durch die Oppositionen von Humanismus und Faschismus gewann, dem also die Kategorien der Holocaust-Debatte noch völlig fremd gewesen sind. Das Wort „Holocaust“ im Sinne von „Judenverfolgung“, „Judenvernichtung“ erscheint übrigens erst am Ende der 1980er-Jahre in der ungarischen Sprache. Damals wurde es noch in seiner lateinischen Form mit ,c' geschrieben, und erst nach 2000 wurde es auch orthographisch völlig „eingebürgert“, d.h. durch ein ,k' umgeschrieben. Dieser Begriffsrahmen, in den sich die Dichtung von Radnóti fast einwandfrei einbetten ließ, machte es möglich, die traumatische Verganheit universalisierend, ohne jeglichen Bezug auf die realen Erfahrungen der zersplitterten ungarischen Gesellschaft, auf die Verantwortung der staatlichen Behörden, der katholischen Kirche, der einzelnen Personen, auf die Notwendigkeit der Untersuchung der herrschenden Diskursformen nach dem ersten Weltkrieg neu zu konzipieren. Dieser unempfindliche Universalismus war der Grund für den zweiten Beitrag der frühen Radnóti-Rezeption zu einer mangelhaften Vergangenheitsbewältigung. Er bestand in der Neubildung einer zukunftsbezogenen nationalen Gemeinschaft, der es anscheinend gelang, über das Trauma der 
Vergangenheit ohne eine Trauerarbeit, ohne die Erweckung des lahmgelegten Mitgefühls hinwegzukommen. Wir müssen uns mit der Tatsache auseinandersetzen, dass die Lektüre der Dichtung von Radnóti Bestandteil eines fast verstummten Zeitalters war, das keine realistische Sprache zur Vergangenheitsbewältigung und zu einer mitbürgerlichen Solidarität ausarbeiten konnte.

Der zentrale Punkt dieser Rezeption war die Interpretation des vielzitierten Gedichtes „Ich kann nicht wissen“ (Nem tudhatom), das sowohl von Franz Fühmann als auch von Markus Bieler ins Deutsche übertragen wurde. Das patriotische Gedicht entstand Ende des Jahres 1943 und Radnóti las es in der Silvesternacht für seine Freunde. Im Mai davor hatte sich Radnóti von Sándor Sík, Prior des Piaristenordens und ehemaliger Professor Radnótis, taufen lassen. In der zweiten Hälfte der 1930er-Jahre und in den ersten Jahren des zweiten Weltkriegs haben mehrere wichtige ungarische Dichter, unter anderem Mihály Babits - in seinem Gedicht „Neunzehnhundertvierzig“ (Ezerkilenszáznegyven), oder Attila József in den Gedichten „Mein Vaterland“ (Hazám) und „An der Donau“ (A Dunánál) versucht, humanistisch-patriotische, aber keineswegs nationalistische Diskursformen aus der ästhetischen Dimension abzuleiten, d.h. alternative Modelle für die Narrativität des Nationalbewusstseins zu liefern, die zum Teil die staatlich verbreiteten postfeudalistischen Diskursformen herausforderten, zum Teil aber auch Wege gesucht haben, die althergebrachten romantischen Diskursformen neu zu konzipieren. Dieser letzte Aspekt ist deshalb unumgänglich, weil die begrifflichen-gefühlsmäßigen Bezugsrahmen in einer halbwegs modernisierten Gesellschaft sowohl für die konservativen als auch für die modernisierenden Konzepte immer noch durch romantische Traditionen vorgeschrieben waren.

Die ersten Zeilen des besagten Gedichts von Miklós Radnóti geben persönlich-familiäre Akzente der romantisch-nationalistischen Formulierung des Gedichts „Mahnruf“ von Mihály Vörösmarty ${ }^{8}$ wieder, in dem die Heimat als eine vorgegebene Macht mit zwingender Kraft den Rahmen für das persönlichen Leben setzt.
Hazádnak rendületlenül
Légy híve, oh magyar;
Bölcsőd az s majdan sírod is,
Mely ápol s eltakar.
Von Lieb und Treu zum Vaterland
bleib, Ungar, stets erfüllt.
Es gibt dir Kraft, und wenn du stürzt, den Hügel, der dich hüllt.

Bei der deutschen Übersetzung von Hans Leicht bleibt das wichtigste diskursbildende Element ausgespart. „Vaterland“ wird bei Vörösmarty mit der Wiege 
und dem Grab, in den weiteren Strophen mit dem Boden identifiziert. Radnótis Gedicht bekräftigt diesen Diskurs in einer Zeit, in der seine Aspiration, als traditionsgebundener Nationaldichter die romantischen Diskursformen zu subjektivieren, mit seinem persönlichen Schicksal in scharfen Konflikt geriet, der scheinbar unreflektiert verstummt, aber desto schrillender im Hintergrund des Gedichts bleibt.

Ich kann nicht wissen, was dem andern dieses Stückchen Land in Flammen hier bedeutet. Mir ist es Vaterland, für mich ist es die Welt, drin die Kindheit weit sich wiegte, ich wuchs aus ihm, ein Reis, das an den Stamm sich schmiegte, und hoffe, daß mein Leib dereinst in dieses Erdreich sinkt. ${ }^{9}$

Als diese Zeilen enstanden, war Radnóti schon zweimal als jüdischer Zwangsarbeiter einberufen und gesetzmäßig aus der Gemeinschaft der Nation ausgeschlossen worden. Er nahm in seinem Gedicht dennoch die Perspektive eines Mitglieds der politischen Gemeinschaft auf, und weil er es zugleich nicht war, weil er sich zur selben Zeit als Opfer äußerte, konnte er die Gemeinschaft der Nation von den uneingestandenen, noch unerschlossenen Sünden entlasten. An diesem Punkt wechselt die Perspektive des Gedichts. Das Familiäre und das Persönliche, die dem Bekenntnis der nationalen Identität als eine reflektierte Zugehörigkeit zum Ort und zur Landschaft eine neue Erfahrungsform verliehen, wird hier eingebüßt. Stattdessen kehrt die alte, in den Ideen der kollektiven Sünde und Sühne verankerte Ideologie des ungarischen Nationalbewusstseins zurück, und zwar so, dass die Sünden sofort durch eine vorschnell geäußerte Aufklärung vergeben werden:

Gewiß, wie andre Völker sind wir schuldig ebenso, wir wissen, wann wir sündigen, womit und wie und wo, doch gibt's auch Bauern, ohne Schuld, Dichter, und zart beschirmt den Säugling, von dem ersten Anhauch der Vernunft gefirmt, sie keimt in ihm, er hütet sie in finsteren Kellern treu bis einst der Friede unserm Land ein Zeichen prägt aufs neu und hell die neue Jugend spricht zum grabentstiegnen Volke.

Das Wort „Vernunft“ stammt hier aus dem Diskurs des antifaschistischen Intellektualismus. Nach dem Krieg haben sich viele Kommentare darauf konzentriert, wie wenig es der europäischen Hochkultur gelungen ist, sich in der Nazizeit der klaren Verletzungen ihrer eigenen Werte zu widersetzen. ${ }^{10}$ Mit Geoffrey Hartman müssen wir aber festhalten, dass wir durch unser Wissen irgendwie damit ,vergiftet" sind, einem nervösen und kritischen Denken nicht entgehen zu können ${ }^{11}$, deshalb geschieht es oft nicht nur, dass wir uns mit dieser Haltung eine Niederlage 
zufügen, sondern auch, dass wir geneigt sind, all denjenigen ein schlechtes Zeugnis auszustellen, die mit ihrer Intellektualität eine Niederlage erlitten haben. Mit seiner kulturellen Einstellung konnte Radnóti seine Wörter aus zwei Diskursformen, aus zwei Wörterbüchern, wählen. Das eine war das Wörtebuch der späten Aufklärung, der sich letzten Endes einer prädikativen Ethik bediente. Das Wort „Vernunft“ stammt aus diesem Vokabular. Es war unbedingt geeignet, dem Nationalismus eine neue Verfassung zu geben, die der staatlich verbreiteten Ideologie kritisch gegenüberstand. Intellektuell und politisch war aber dieser Nationalismus mittel- und machtlos. István Vas, einer der Freunde, der dabei war, als Miklós Radnóti das Gedicht „Ich kann nicht wissen“ in jener Silvesternacht las, nannte seine Heimat das literarische Ungarn, die Bücherschrank-Nation. „Te egyetlen hazám, te szépséggel teli! / Nem jelen arcod az, mely szívem élő vágya, / Szerelmem Magyarország, a képzeletbeli, / Arany, Balassi, Zrínyi, kurucok szebb világa."'2 (Menekülő múzsa)

Das andere Wörterbuch, das sich für Radnóti bereitstellte, war das Vokabular der herrschenden postfeudalen Ideologie: voller verschwommener, mythisch-religiöser Vorstellungen über ein ruhmvolles Land in arger Bedrängnis, die keine Möglichkeiten gaben, sich mit der realen Lage von Ungarn in Europa auseinanderzusetzen. Radnóti war wegen seiner katholischen Religiosität auch dieses Wörterbuch nicht fremd. In der ersten Fassung des Gedichts schrieb er in der letzten Zeile: „Breite, Heilige Maria, deine wachenden, himmlischen Schwingen über uns“. Die Frau des Dichters erinnerte sich an eine andere Version: „Breite deine großen Schwingen, Patrona unserer Heimat, über uns“.

Die Freunde, unter ihnen István Vas, Gyula Ortutay, Gábor Tolnai und Tamás Major, die das Gedicht in der Silvesternacht 1943 hörten, zeigten sich darüber entsetzt. Ihre Vorwürfe und Ablehnung haben Radnóti zutiefst gequält. Die letzte Zeile hat sich verändert, der darin eingedrungene mystisch-religiöse Diskurs der herrschenden Staatsideologie hat nachgelassen und für eine dunkle Vorahnung Platz gemacht: „Breite deine Schwingen über uns, wachende dunkle Wolke.“

Eine realistische, anti-idyllische Sprache entsteht in der Dichtung von Radnóti erst in den späten Gedichten, vor allem in denen, die im Notizheft von Bor erhalten geblieben sind. Dieses Heftchen ist ein einzigartiges Medium der Literaturgeschichte. Es vermittelt uns die Handschrift des Dichters als ein persönliches Zeugnis von jenseits aller Grenzsituationen. „Razglednica“, der Gattungsname der letzten Gedichte, bedeutet „Postkarte“. Die Funktion der Vermittlung des Unvermittelbaren, des Verbindens des Unverbindbaren verleiht der Handschrift eine einzigartige Qualität. Die Wörter in diesem Heft haben die Schicksalsereignisse des Martyriums, die Traumata der Beerdigung und der Exhumierung zusammen mit dem Körper des Dichters mitgemacht. Die Zeit kommt in dieser Handschrift zum Stillstand. Die Gedichte erhalten sich als Ablagerung der Zeit. Nur eine Zeile scheint ihre Fähigkeit zur temporalen Bewegung zu behalten: Ein deutscher Satz im vierten Stück des Zyklus. 
Mellézuhantam, átfordult a teste

$\mathrm{s}$ feszes volt már, mint húr, ha pattan.

Tarkólövés. - Így végzed hát te is, -

súgtam magamnak, - csak feküdj nyugodtan.

Halált virágzik most a türelem. -

Der springt noch auf, - hangzott fölöttem.

Sárral kevert vér száradt fülemen.

Szentkirályszabadja, 1944. október 31.

Er, neben dem ich hingestürzt lag, war schon

verrenkt, verspannt, wie Saiten springen.

Genickschuss. - Also, - raunte ich mir zu, -

Geduld bringt jetzt die Rose Tod hervor. -

„Der springt noch auf“, - scholl es über mich hin.

Mir klebte Dreck vermischt mit Blut am Ohr.

(Übersetzung von Markus Bieler)

Dieses Gedicht hat Radnóti neun Tage vor seinem Tod geschrieben. Der ungarische Kader von Radnótis Zwangsarbeitereinheit wurde bei Szentkirályszabadja von deutschen Soldaten abgelöst. Sie erschossen den Freund des Dichters, Ferenc Lorsi. Dieser Moment wird im Gedicht ,verewigt“. Für die Vermittlung des Mordes liefert die deutsche Sprache die Wörter, wodurch die Mordtat von der ungarischen Sprache ferngehalten wird. Ist der Tod auch hier ein Meister aus Deutschland? Nein, er ist auch ein Meister aus Ungarn. Das bleibt im Gedicht dem Ungarischen erspart. Die Fremdheit der Mordtat wird erst in der deutschen Übersetzung verwischt. Das Gedicht kommt auf Deutsch beim Tod, bei der Erde an.

\section{Anmerkungen}

1 Aleida Assmann, Erinnerungsräume. Formen und Wandlungen des kulturellen Gedächtnisses, (München: C. H. Beck, 2003), S. 28.

2 Sigmund Freud, „Konstruktionen in der Analyse”, in: Gesammelte Werke Bd. XVI, hrsg. von Anna Freud, (Frankfurt am Main: Suhrkamp Verlag, 1969), S. 46.

3 Walter Benjamin, Gesammelte Schriften, Bd. IV, 1. (Frankfurt am Main: Suhrkamp Verlag, 1991), S. 400.

4 Aleida Assmann, op. cit. S. 165.

5 Ortutay Gyula, Napló I., (Pécs: Alexandra Kiadó, 2009), S. 371.

6 Ortutay Gyula, Radnóti Mikós - In: Baróti Dezső (Hrsg.): Radnóti Miklós 1909-1944 (Budapest: Magyar Helikon, 1959) S. 7.

7 Bóka László, Tajtékos ég, Magyarok, 2/8. (1946), S. 472-473.

8 Ferencz Győző, Radnóti Miklós élete és költészete (Budapest: Osiris Kiadó, 2005), 622.

9 Übersetzung von Franz Fühmann

10 Geoffrey Hartmann, Der längste Schatten, (Berlin: Aufbau Verlag, 1999) S. 189.

11 Geoffrey Hartmann: ebenda 
12 Du, meine einzige Heimat, du voller Schönheit, das lebendige Begehr meines Herzens ist nicht dein gegenwärtiges Gesicht, meine Liebe ist Ungarn, das vorgestellte, eine schönere Welt von Arany, Balassi, Zrínyi und der Kuruzen.

Open Access. This is an open-access article distributed under the terms of the Creative Commons Attribution 4.0 International License (https://creativecommons.org/licenses/by/4.0), which permits unrestricted use, distribution, and reproduction in any medium, provided the original author and source are credited, a link to the CC License is provided, and changes - if any - are indicated. (SID_1) 